\title{
Experimental evaluation of a telecommunications network along railway lines by ad hoc network technology
}

\author{
K. Seki \\ Railway Technical Research Institute, Japan
}

\begin{abstract}
There are some cases when we would like to construct tentative communications systems easily along railway lines even if they cannot fully guarantee to satisfy transmission qualities. For example, we need to grasp situations of disasters or accidents which have happened at the place where not enough transmission bandwidths are secured or the transmission system itself has been damaged. Ad hoc network technologies are a candidate for constructing such tentative networks. Their standardizations are in progress and some commercial products are emerging. We carried out an experiment using commercial products which implemented a standardized protocol and constructed a network by wireless LAN, and confirmed that the network was easily constructed but frame loss rates and time to change routes when devices broke were insufficient for some cases. We, therefore, tentatively made telecommunications nodes which used both wired LAN and wireless LAN. We confirmed that a satisfying performance was obtained until about three wireless hops even if only wireless LAN was used, and transmissions continued even when a cable or the node was damaged.
\end{abstract}

Keywords: ad hoc network, image transmission.

\section{Introduction}

Railway telecommunications systems, such as train radio, are important infrastructures which support train operations. After they are minutely designed in order to satisfy requirements such as coverage areas and quality of services, fixed equipments such as wireless base stations, exchanges, and wired transmission lines which connect between them are installed. The systems can, therefore, maintain the required transmission qualities for a long time once they 
are installed, but a lot of time and costs are needed until they start their operations. On the contrary, there are some cases when we would like to construct tentative communications systems easily along railway lines even if they cannot fully guarantee to satisfy transmission qualities. For example, we need to grasp situations of disasters or accidents which have happened at the place where not enough transmission bandwidths are secured or the transmission system itself has been damaged. Ad hoc network technologies are a candidate to construct such tentative networks. Their standardizations are in progress and some commercial products are emerging.

In this paper, we outlined the ad hoc network technologies and showed results of experiments in our Institute which were carried out imaging to apply the technologies to transmission systems along railway lines and pointed out some issues. Then, we introduced our tentatively produced system to resolve the issues by using both wired and wireless channels and reported that expected characteristics were obtained from our experiments in our Institute.

\section{Examples of usages of telecommunications systems along railway lines}

In this paper, we are assuming to construct telecommunications network systems along railway lines applicable to the following purposes.

\subsection{Tentative telecommunications systems}

This is a network to connect cameras at accidental points with the nearest station, or handy terminals of maintenance workers with the nearest station when maintenance works are taking place where telecommunications infrastructures are insufficient. As this network has to be set up instantaneously, it is required to configure the network with the least operations.

\subsection{Telecommunications systems with redundancy}

This is a network to connect devices such as supervisory cameras and sensors located at places where some omens of disasters are recognized with the nearest station for a relatively long period. The telecommunications system is required not only to be set up instantaneously but also to continue transmissions even if telecommunications devices or transmission media are damaged.

\section{Ad hoc network technology}

\subsection{Outline of ad hoc network technology}

Ad hoc network technology is thought to be suitable for constructing networks for the purposes described in Section 2. We briefly explain the ad hoc network technology.

Ad hoc network is a network which is constructed by terminals only without any telecommunications infrastructure such as base stations, and its purpose is to 
construct a network where no telecommunications infrastructures exist. So the terminals have to possess capabilities to find a route to destination terminals even if telecommunications environment changes by the terminals' movements, and to relay data frames between the other terminals. In general, transmission media used for ad hoc networks are wireless such as wireless local area networks (WLAN) and Bluetooth because they are suitable for tentative constructions of networks. On the other hand, networks constructed by the nodes which have capability of multi-hop functions (functions to use other terminals as relay nodes) are sometimes called ad hoc networks also even if they use wired transmission media or fixed equipment as well as wireless ones.

IETF (Internet Engineering Task Force) are working for standardizations of telecommunications protocols to realize ad hoc networks. MANET (Mobile Ad hoc Networks) group in the IETF is now standardizing two protocols called DYMO (Dynamic MANET on Demand) [1] and OLSRv2 (Optimized Link State Routing version 2) [2].

Some commercial products are off the shelf. Figure 1 (a) is Meshrouter. This product makes a protocol based on AODV (Ad hoc On-demand Distance Vector) [3] ,which is an original protocol of DYMO, act on IEEE 802.11g wireless LAN channels, and has wired and wireless LAN interfaces to connect devices such as PCs and the Internet. Figure 1 (b) is an experimental device called Fieldserver, which has the same ad hoc network card as Meshrouter and an IP camera. This was originally developed to supervise the growth of crops at farms.

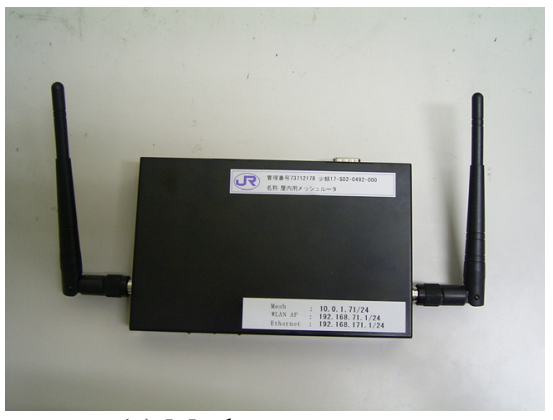

(a) Meshrouter

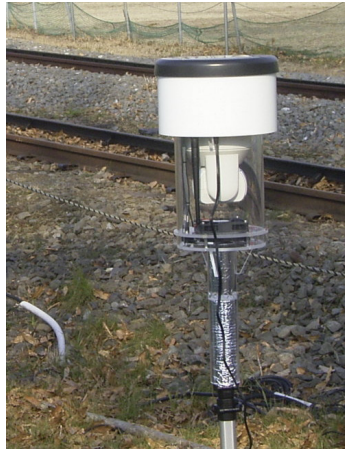

(b) Fieldserver

Figure 1: $\quad$ Examples of commercial products.

\subsection{Experiments using the commercial products}

We carried out an experiment with the commercial products in our Institute to measure their performance, assuming telecommunications systems along railway lines described in Section 2. We arranged six Meshrouters along a test railway line at our Institute (Figure 2). After we set basic information such as their own IP addresses and used wireless channels to the routers, the routers could configure an ad hoc network after about one minute and we confirmed that these were applicable as a simple method to construct a network. 


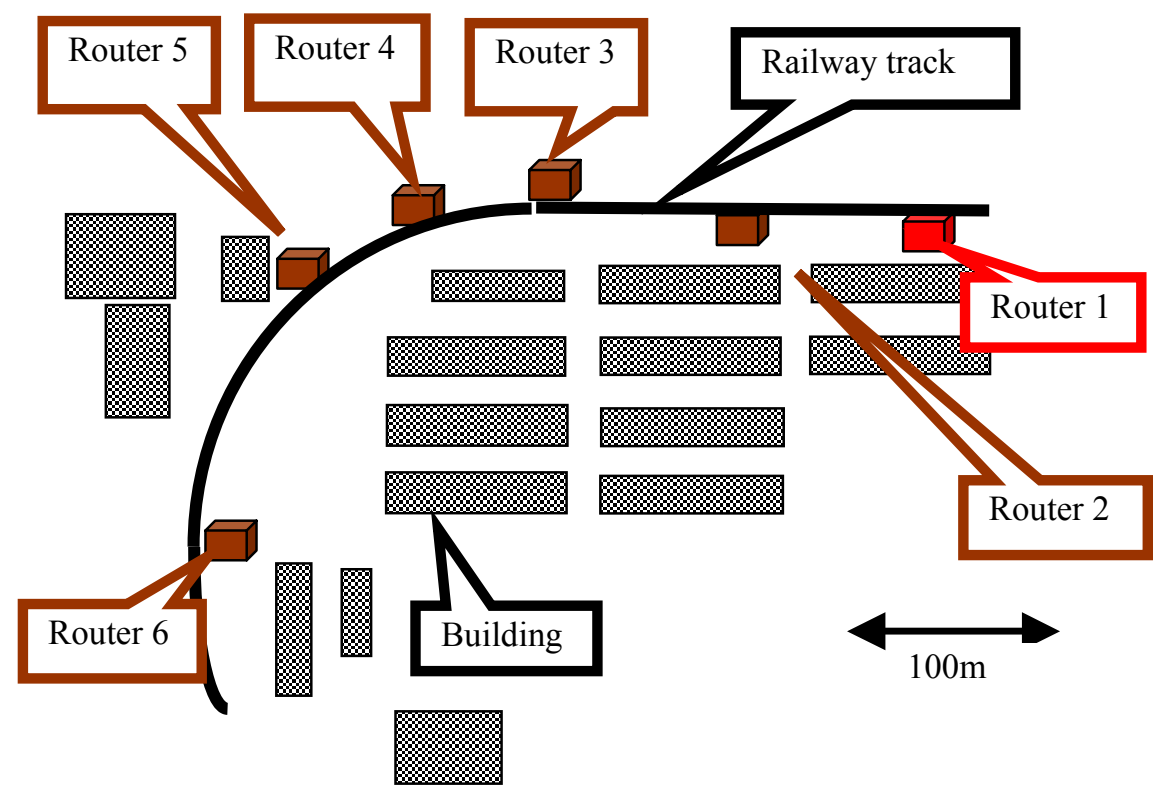

Figure 2: $\quad$ Layout of routers.

Experimental results were as follows.

(1) Frame loss rate and roundtrip time

We connected one PC to the router 1 and another PC to one of the rest routers, and measured frame loss rate and roundtrip time by a ping command. Table 1 shows the results of the experiment.

Table 1: $\quad$ Frame loss rate and average roundtrip time.

\begin{tabular}{|c|c|c|}
\hline Interval & Frame loss rate (\%) & Average roundtrip time (ms) \\
\hline router 1 and 3 & 6 & 6 \\
\hline router 1 and 4 & 11 & 12 \\
\hline router 1 and 5 & 0 & 10 \\
\hline router 1 and 6 & 10 & 70 \\
\hline
\end{tabular}

In general, as the distance between two PCs increases, the frame loss rate and the average round trip time increase. We infer that the reason why the transmission quality between router 1 and 5 is better than those of router 1 and 3 and router 1 and 4 is because the route in the former case was router 1-2-4-5 and in the latter cases router 1 and 3 or 4 were directly connected. In the former case, each wireless links thought to be short and stable though the hop counts were larger. In any case, there were the situations where the transmission quality was not satisfying.

(2) TCP throughput

We connected one PC to the router 1 as a ftp server and another PC to one of the rest routers as a ftp client, and measured throughput as TCP throughput. 
Table 2 shows the results of the experiment. In general, the lower the frame loss rate described in (1) is, the higher the throughput becomes. Non-transmission situations existed because of waiting for retransmission in the cases of low throughput.

Table 2: $\quad$ TCP throughput.

\begin{tabular}{|c|c|}
\hline Interval & TCP throughput $(\mathrm{kByte} / \mathrm{sec})$ \\
\hline router 1 and 3 & 221 \\
\hline router 1 and 4 & 167 \\
\hline router 1 and 5 & 269 \\
\hline router 1 and 6 & 96 \\
\hline
\end{tabular}

We replaced the router 6 with a Fieldserver which transmitted images to the router 1 at the maximum frame rate of 15 frames / second and measured throughput between the router 1 and the router 4 . It degraded to about $100 \mathrm{kByte}$ / sec.

(3) Route changes

While we executed ping commands from a PC connected to the router 5 to a PC connected to the router 1 , we powered off the router 4 which was acting as a relay. In this case, one or a few data frames were lost, then the routers reestablished another appropriate route after about one second, and transmission restarted.

\subsection{Issues recognized from the experiment}

The commercial products based on the standardized protocol select a suitable route from a source node to a destination node at first, and then transmit a single copy of each data frame. This is a desirable behaviour in general because bandwidths of wireless channels are narrow and their efficient use is the most important. In railway operation, however, there are cases where reliability of transmissions, and robustness against damages of devices and cables and interferences to wireless channels are more important than efficiency. So the commercial products are required to be improved regarding frame loss rates and route change time, though they are excellent in constructing a network easily.

\section{Trial products for telecommunications systems along railway lines}

As mentioned above, networks by the existing products sometimes lack continuity and reliability of transmissions in some applications. The main reason is that they use only wireless channels. So we decided to produce telecommunications nodes tentatively which use both wired and wireless channels at the sacrifice of the simplicity of constructions when reliability of transmissions is required, and experimented on their performance at our Institute. 


\subsection{Specifications of the tentatively produced telecommunications nodes}

The telecommunications nodes have two wired LAN interfaces (100BASE-T) and two wireless LAN interfaces (IEEE 802.11b). Figure 3 shows an appearance of the telecommunications node. This is a general-purpose small size Linux box which has two wired LAN interfaces and two PCMCIA slots in which we insert wireless LAN cards.

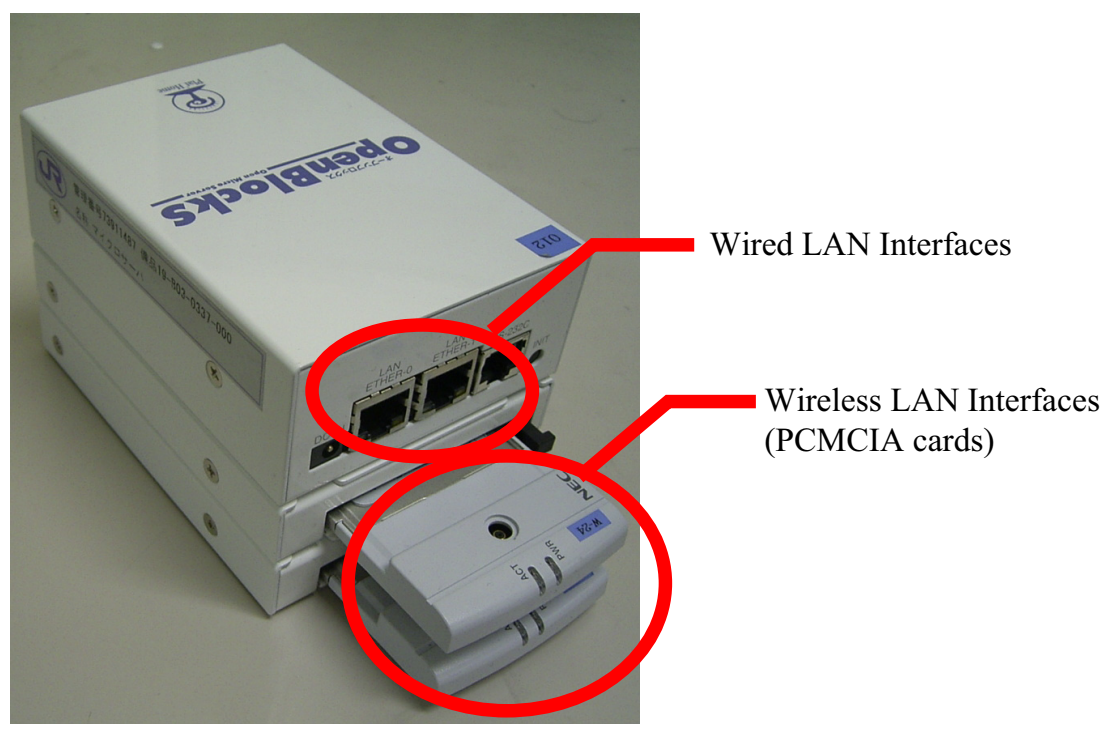

Figure 3: Appearance of telecommunications node.

Three types of the telecommunications nodes exist, that is, a gateway node, a camera node, and a relay node (Table 3). These nodes construct an ad hoc network, and devices such as PCs and cameras which utilize the network are connected to the gateway node or the camera node via one of the wired LAN interfaces of the nodes. As a result, three interfaces (one wired and two wireless) of the gateway node and the camera node and four interfaces (two wired and two wireless) of the relay node are used to construct the ad hoc network. Figure 4 illustrates an example of connections between the nodes.

\subsection{Basic behaviour of the nodes}

A gateway node periodically transmits "Hello" frames from three interfaces (one wired and two wireless) of the node, which are used for the ad hoc network simultaneously. A relay node retransmits the received frames in the following manner.

(a) If it receives the frame from a wireless interface, it retransmits the frame to the same wireless interface. 
Table 3: $\quad$ Types of telecommunications nodes.

\begin{tabular}{|l|l|}
\hline Type & Explanation \\
\hline $\begin{array}{l}\text { Camera } \\
\text { node }\end{array}$ & $\begin{array}{l}\text { An IP camera or a PC is connected to this node via wired LAN. } \\
\text { The node transmits images from the camera to a gateway node. } \\
\text { More than one nodes of this type may exist in a network. }\end{array}$ \\
\hline $\begin{array}{l}\text { Gateway } \\
\text { node }\end{array}$ & $\begin{array}{l}\text { A PC to display images from the camera nodes is connected to } \\
\text { this node via wired LAN. Only one node of this type exists in } \\
\text { a network. }\end{array}$ \\
\hline Relay node & $\begin{array}{l}\text { This node relays data frames between the camera node and the } \\
\text { gateway node. More than one nodes of this type may exist in a } \\
\text { network. }\end{array}$ \\
\hline
\end{tabular}

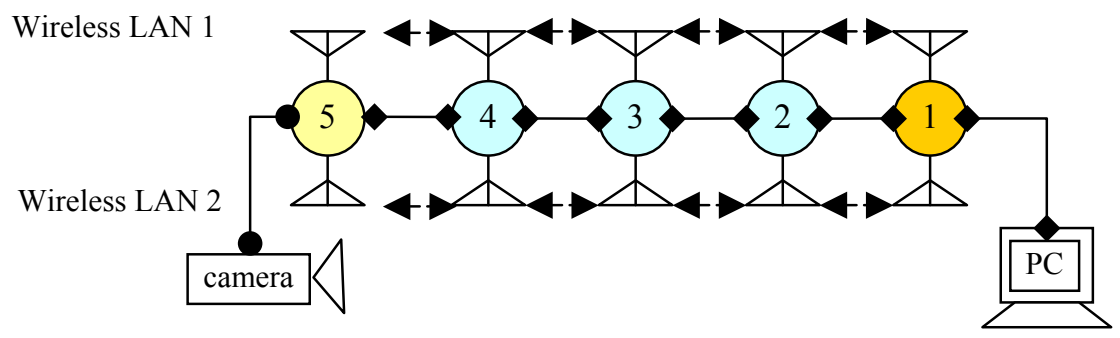

Node $1=$ Gateway node, Node 2,3,4=Relay node, Node 5=Camera node

Figure 4: $\quad$ Example of ad hoc network.

(b) If it receives the frame from a wired interface, it retransmits the frame to the other wired interface.

The camera node which receives the "Hello" frame can recognize an IP address of the gateway node.

Data frames such as images are transmitted in the same manner. If all interfaces of the nodes are active, the gateway node receives at most the same three data frames from the camera node. The gateway node transmits the frame which arrives at the node first to the PC connected to the node via the wired interface, and discards the rest two frames which arrive later. As a result, the data frames via the wired channel which normally has the least delay are adopted. If the wired channel is damaged, there will be no time when communications are unavailable, as the data frames via the wireless channel can be used instantaneously.

\subsection{Experiments at our Institute}

\subsubsection{Outline of experiments}

We arranged twelve nodes (one gateway node, two camera nodes, nine relay nodes) at our Institute (Figure 5). Figure 5 also shows node identifiers (ID) which are used to refer to the nodes.

Nodes 1 through 8 are serially connected by LAN cables. 


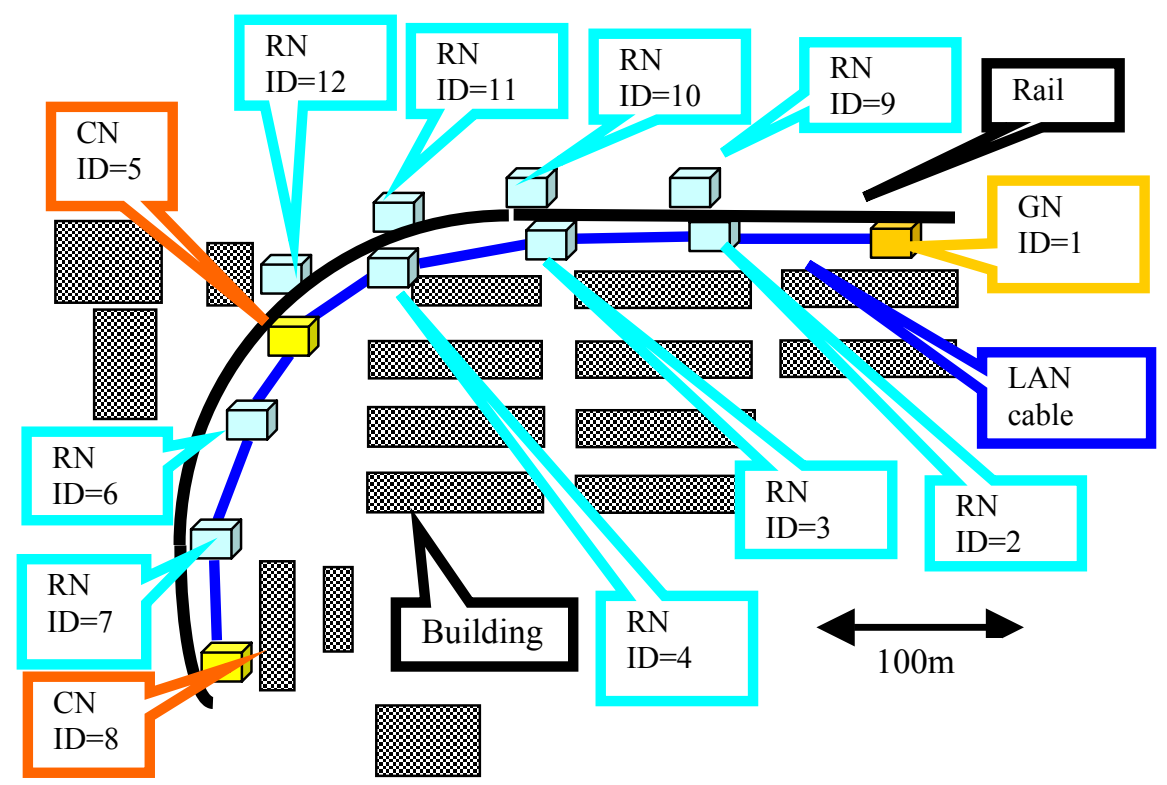

GN: Gateway Node, CN: Camera Node, RN: Relay Node

Figure 5: $\quad$ Layout of communications nodes.

\subsubsection{Experimental results}

(1) Frame loss rate and roundtrip time

We connected one PC to the gateway node and another PC to one of the camera nodes (ID $=5$ or 8 ), and measured frame loss rate and roundtrip time by a ping command while both wired LAN and wireless LAN were active. We measured them while the LAN cables were disconnected and only wireless LAN was active. Table 4 shows the results of the experiment. In the Table 4 , "Node ID" column designates the node ID of the camera node to which the PC was connected.

The transmissions were stable with low roundtrip time of about $0.75 \mathrm{~ms}$ per link while wired LAN was available. On the other hand, when only wireless LAN was available, no frame loss was observed with lower quality of round trip time than wired LAN transmission between the node 1 and the node 5, but high frame loss rate was observed between the node 1 and the node 8 .

Table 4: $\quad$ Frame loss rate and average roundtrip time.

\begin{tabular}{|c|c|c|c|}
\hline Node ID & $\begin{array}{c}\text { Transmission } \\
\text { media }\end{array}$ & $\begin{array}{c}\text { Frame loss rate } \\
(\%)\end{array}$ & $\begin{array}{c}\text { Average roundtrip time } \\
(\mathrm{ms})\end{array}$ \\
\hline 5 & Wired LAN & 0 & 3 \\
\hline 5 & Wireless LAN & 0 & 35 \\
\hline 8 & Wired LAN & 0 & 5 \\
\hline 8 & Wireless LAN & 2.2 & 37 \\
\hline
\end{tabular}


(2) TCP throughput

We connected one PC to the gateway node and another PC to one of the camera nodes (ID $=5$ or 8 ), and measured TCP throughput by iperf utility. We also measured it in a laboratory in the situation where only the gateway node and the camera node existed. Figure 6 shows the results of the experiment. In Figure 6 , horizontal axes are the number of the links for wired transmission or the number of hops for wireless transmission.

We can say that a fairly high throughput is obtained even via seven links when wired channel is available, and the throughput over wireless LAN of three hops is about $230 \mathrm{kbps}$. The reason why the throughput is lower than the result of Section 3 is because the nodes use IEEE 802.11b wireless LAN of the maximum bit rate $11 \mathrm{Mbps}$, while the commercial products described in Section 3 use IEEE $802.11 \mathrm{~g}$ wireless LAN of the maximum bit rate $54 \mathrm{Mbps}$.

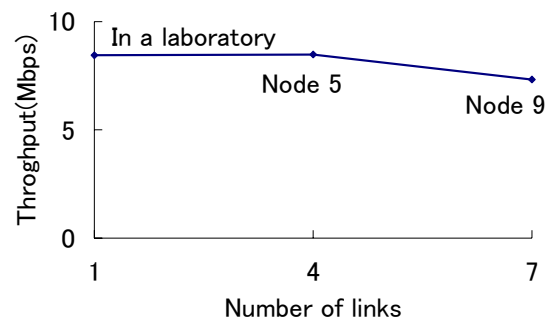

(a) Wired transmission

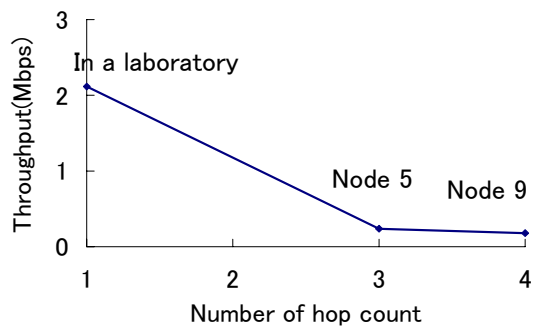

(b) Wireless transmission

Figure 6: $\quad$ TCP throughput.

(3) Image transmission

We connected a PC to the gateway node and an IP camera to one of the camera nodes (ID $=5$ or 8 ). The IP camera transmitted MPEG format images to the PC. We subjectively evaluated the receiving images displayed by a viewer on the PC.

When we connected the LAN cables, transmissions of a resolution of $640 \mathrm{x}$ 480 pixels and a frame rate of 15 frames / second were possible from either the node 5 or the node 8 . On the other hand, when we disconnected the LAN cables, transmissions from the node 8 were unpractical and transmissions of a resolution of $240 \times 180$ pixels and a frame rate of 15 frames / second were acceptable from the node 5 .

While we were transmitting images over wired LAN from the node 5, we disconnected a LAN cable between the node 3 and the node 4 . However, we confirmed that receiving images was continuously displayed on the viewer without affected by the damage of the cable.

Then, we made two wireless channels of the node 1 broken down alternately. We confirmed that this did not affect the receiving images. So we can say this transmission system has robustness against damages of cables and node interfaces, and even in the situation of damages of them, has a transmission capacity to transmit images of an appropriate quality. 


\section{Conclusion}

A tentative telecommunications network along railway lines which can be constructed easily is required in some cases. Even in these cases, it is also required to enhance transmission reliability and robustness sometimes. The standardized ad hoc routing protocol and commercial products based on it are adaptable to cases when instant of network construction is required, but they are insufficient for cases which require more reliability. The tentative products we developed are applicable to both situations because they can use not only both wired and wireless channels to enhance transmission reliability but also only wireless channels to construct networks quickly. From our experiments in our Institute, we verified that our products had good performance until three wireless hops and transmissions continued even when cables or nodes were damaged.

We are considering to enhance our products by making them capable to treat more wireless channels or wireless systems such as IEEE 802.11a.

This work is financially supported in part by the Japanese Ministry of Land, Infrastructure and Transport.

\section{References}

[1] IETF: Dynamic MANET On-demand (DYMO) Routing, Internet-Draft, 2008

[2] IETF: The Optimized Link State Routing Protocol version 2, Internet-Draft, 2008

[3] IETF: Ad hoc On-Demand Distance Vector (AODV) Routing, RFC3561, 2003 